\title{
Lumbar disk 3D modeling from limited number of MRI axial slices
}

\author{
Asma'a Al-Mnayyis ${ }^{1}$, Sanaa Abu Alasal ${ }^{2}$, Mohammad Alsmirat ${ }^{3}$, Qanita Bani Baker ${ }^{4}$, Shadi AlZu'bi $^{5}$ \\ ${ }^{1}$ Department of Clinical Sciences-Radiology Division-MSK Radiologist, Faculty of Medicine, \\ Yarmouk University, Jordan \\ ${ }^{2,3,4}$ Department of Computer Science, Jordan University of Science and Technology, Jordan \\ ${ }^{5}$ Computer Science Department Al Zaytoonah University of Jordan, Jordan
}

\begin{abstract}
Article Info
Article history:

Received Dec 6, 2019

Revised Feb 10, 2020

Accepted Feb 24, 2020

\section{Keywords:}

3D modeling

Disk herniation

Isosurface

Marching cubes

MRI

ABSTRACT

This paper studies the problem of clinical MRI analysis in the field of lumbar intervertebral disk herniation diagnosis. It discusses the possibility of assisting radiologists in reading the patient's MRI images by constructing a 3D model for the region of interest using simple computer vision methods. We use axial MRI slices of the lumbar area. The proposed framework works with a very small number of MRI slices and goes through three main stages. Namely, the region of interest extraction and enhancement, inter-slice interpolation, and 3D model construction. We use the Marching Cubes algorithm to construct the $3 \mathrm{D}$ model of the region of interest. The validation of our 3D models is based on a radiologist's analysis of the models. We tested the proposed 3D model construction on 83 cases and We have a 95\% accuracy according to the radiologist evaluation. This study shows that $3 \mathrm{D}$ model construction can greatly ease the task of the radiologist which enhances the working experience. This leads eventually to a more accurate and easy diagnosis process.
\end{abstract}

Copyright $(02020$ Institute of Advanced Engineering and Science. All rights reserved.

\section{Corresponding Author:}

Mohammad Alsmirat,

Department of Computer Science,

Jordan University of Science and technology,

Ar Ramtha 3030، Ar-Ramtha, Jordan.

Email: masmirat@just.edu.jo

\section{INTRODUCTION}

Disk herniation is one of the main causes of low back pain. It is a class of disk related degeneration defined as a local displacement of disk ingredients toward the posterior normal margins of the inter-vertebral disk [1]. In most cases, herniation occurs in the lower lumbar area, and it is detected by the presence of pain in the lower back area extending towards the legs [2,3]. Herniation is usually categorized in several ways [4].

- Based on the type such as protrusion, extrusion, and sequestration.

- Based on the location such as central, posterolateral (right or left), and foraminal (right, left orboth).

- Depending on whether it has compress on or displace the nerve root, which is a cause of severe pain.

Magnetic resonance imaging (MRI) is one of the routines conducted in the management and the diagnosis of diseases such as spinal stenosis, disk herniation, or tumors [4, 5]. MRI uses magnetic fields to obtain a set of multiplanar images of the body organs. Those anatomic details of the 3D organ are presented as a set of 2D parallel cross-sectional (axial), sagittal or coronal images [6]. MRI scans are the most widely used imaging modality for examining the anatomy and the physiology of the body despite being the most expensive one [7]. Mostly, the automatic diagnostic research that is based on clinical MRI data uses only the sagittal views [8-10], even though radiologists scan both sagittal and axial planes to make their final diagnosis [4]. 
In general, reading the MRI images is a challenging process and need time and effort from both radiologists before the case can be diagnosed. The aforementioned challenges motivated researchers to study converting medical images into quantitative measurements [4, 11-13]. In another direction, researchers studied the possibility of building 3D models from those medical image [2, 13-16, 9, 10] to make reading the images easier for the specialists.

In this paper, we are planning to build a 3D model for the disks in the lumbar area from a set of stacked 2D axial MRI slices. The area that we are studying is the area between the lumbar vertebrae 4 and 5 (L4/L5 disk level). Our method can be used to build a 3D model from a very limited number of axial MRI slices as it is usually very difficult to get more slices in this area. The 3D model that we built is used in visualizing the disk to assist the specialist in the manual diag-noses of the studied cases. By performing the analysis and the evaluation of the 3D models, the radiologist finds that it is easier to reach the diagnosis based only on the 3D model in $95 \%$ of the cases. The rest of the paper is organized as follows. In the next section, we review the previous works in the field of this study, and section 3 discuses the dataset we use in our experiments. The proposed 3D model construction is detailed in section 4. Finally, the results are presented in section 5 .

\section{LITERATURE REVIEW}

Many studies can be found in the literature that deal with medical 3D model construction but non of them was done on a limited number of MRI axial view scans of the disks in the lumbar area. For the 3D modeling approaches in tumor field, Arakeri et al., [2] developed a 3D construction and quantification method for assisting the physicians in surgical planning and volume computation of brain tumors. They reconstructed a 3D brain tumor from a given set of 2D MRI slices of the brain by developing methods for segmentation, inter-slice interpolation, mesh generation and simplification. Slices that contain tumor were extracted from a given set of axial slices of the brain. They used a centroid alignment technique and interpola- tion in enhancing the shape which helped in estimating the missing slices accurately between the chosen slices. Their experimental results showed that the proposed 3D construction approach can generate an accurate 3D model in less time, and it can assist the radiologist to identify the stage of the tumor and the treatment planning.

Ali et al., [13] have developed a fully auto, easy, and effective segmentation technique for detecting and extracting the tumor region from three sequential T1-weighted MRI slices. These segmentation results were used in order to reconstruct a 3D image for the tumor region. Then the resulted 3D tumor was inserted in $3 \mathrm{D}$ contour image of the brain. The relative size of the tumor has been measured with respect to the brain size. This work showed that the proposed method could be used in detecting and extracting the brain tumor in the MRI images. Also, they showed that their method can be incorporated in longitudinal studies for analyzing the evolution of the tumors and their impact on the surrounding structures. Furthermore, their results showed that their model can be used for the diagnosis, the treatment planning, the therapeutic monitoring, surgery, and pathological brain modeling.

Kızılgo“z et al., [17] have conducted a comparison between three-dimensional turbo spin-echo sequences versus conventional two-dimensional turbo spin-echo sequences in the diagnosis of lumbar interver- tebral discs. They concluded that the 3D features are very usefull and it use is comparable to the use of the conventional MRI. A framework for 3D surface-mesh-based segmentation of healthy and herniated disks of the lumbar spine over sagittal planes was proposed by Haq et al., [9]. In addition, this paper presented a proof of concept of their segmentation method as part of a processing pipeline for anatomical models input to interactive surgery simulation, especially of a discectomy procedure, in conjunction with the SOFA-based simulation that captures spine pathology with fidelity. Ground truth model is made by SOFA. The mean absolute shape distance, MASD, (in $\mathrm{mm}$ ) and absolute standard deviation of all errors (in $\mathrm{mm}$ ), absolute mean square distance MSD (in $\mathrm{mm}$ ), the Hausdorff distance (in $\mathrm{mm}$ ) and DICE similarity coefficient comparison metrics have been calculated to compare the quality of their segmentation approach with ground truth. Where the Hausdorff distance is the maximum surface distance between two surface meshes and quantitatively represents a measure of the worst segmentation error.

\section{DATASET}

The dataset used in this work is taken from King Abdullah University Hospital (KAUH) - Irbid, from the radiology department (https://bit.ly/2BEWkwM). The same raw dataset is used to extract the imaes used in $[7,18,19]$. From the raw dataset, the cases that have multiple slices per disk are chosen because they can be used in 3D construction of the disk area. A radiologist helped us in the annotation and clarification of 
the cases. She also built a ground truth for the studeid images. And finally, she validate the results of the 3D construction of the cases.

Each patient is involved in only one imaging session. Imaging results contains T1 and T2 weighted sequences in the sagittal plane (series from right to left) in addition to axial T1 and T2 slices that are cutting the body transversely. For the lumbar area, the number of slices in the sagittal view is between 11 and 13 . In the axial view, the number of slices is between 10 and 20 and there are 5 cuts cover the intervertebral disk between every two vertebrae (spinal segment). These 5 cuts are positioned at the lower endplate of the upper vertebra, upper part of the disk, mid of the disk, bottom of the disk and at the upper endplate of the lower vertebra. These locations are the best to describe the intervertebral disk shape.

The thickness of each slice is $4 \mathrm{~mm}$ in average, but this does not mean that the space between slices is $4 \mathrm{~mm}$. The distances between slices are different and are determined by the specialist to take slices in certain places that appear necessary for the diagnostic process. These places are usually determined on a sagittal scout images that start from left to right so that the spine is cut longitudinally to show the spinal cord, the neural canals, and surrounding tissues. We can notice from Figure 1 that there is a big distance between every two slices. This distance illus- trates the shrinking and the disappearance of some tissues located between those slices. Hence, we found that it is better to generate artificial slices between each two. The artificial slices are generated using interpolating adjacent original slices.
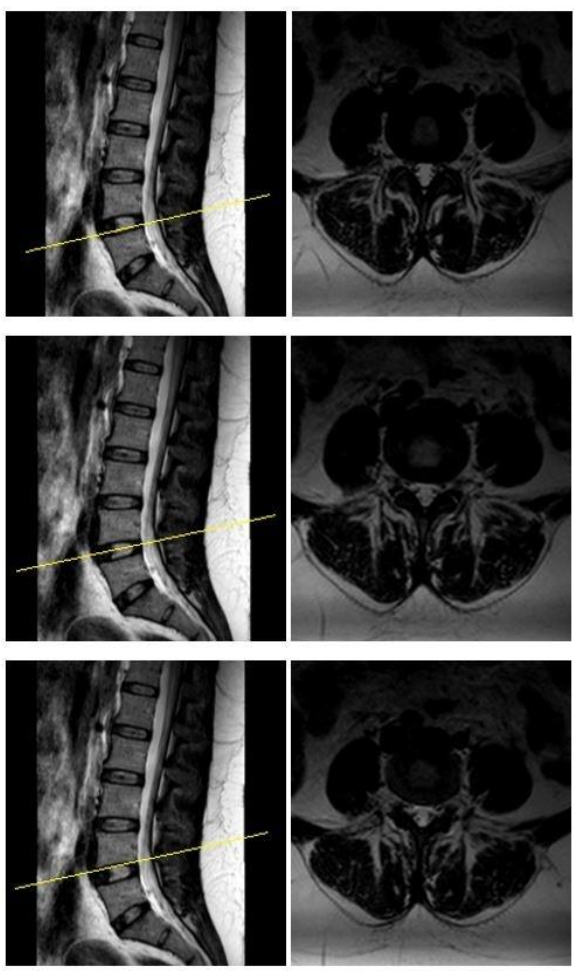

Figure 1. Positions of the MRI cuts lower, middle and upper the disk

In this way, we can build a model that simulates the shape of the area on which the diagnosis is applied. Our study includes only the axial T2 weighted image that shows a high signal intensity for the structures with higher water content such as the cerebrospinal fluid CSF and the nucleus pulposus of the disk [4]. The area to be diagnosed in each slice is the margin that lies between the posterior of the lumbar disk and the interior of the thecal sac, this area is illustrated in Figure 2.

In the axial view, the healthy disk looks like a circle with black margins and gray colored middle. The thecal sac area looks like a triangle; with holes indicate the passage of the spinal nerves through it. For lateral neural foramen (NF), they appear clearly, as they allow the traversing nerves to exit from the sides of the thecal sac to supply the organ that they are related to [20]. On the other hand, in disk herniation cases, the disk appears to be bulged out of the NF fiber going out to its posterior side that touches the anterior area of the thecal sac. This bulging appears in the form of a black area extending posteriorly to the anterior part of the thecal sacand NF. 


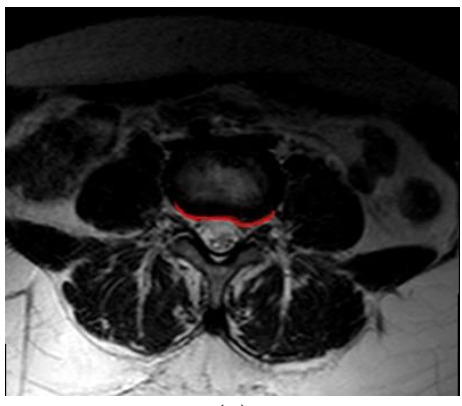

(a)

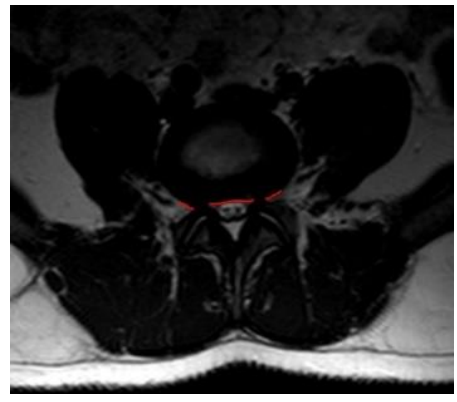

(b)

Figure 2. Red lines that indicates the area of interest, (a) normal case, (b) abnormal case

We obtain 83 cases divided into 45 abnormal cases and 38 normal cases. The radiologist examined these cases to build a ground truth for them. In the abrormal cases, 30 cases are diffus, 15 cases are foraminal (one Left-foraminal, one Right-foraminal and 13 Bi-foraminal). This ground truth is detailed in Table 1.

Table 1. Training cases distribution of classes

\begin{tabular}{cc}
\hline Class type & No. of cases \\
\hline Normal & 38 \\
Diffuse & 30 \\
Bi-foraminal & 13 \\
Left foraminal & 1 \\
Right foraminal & 1 \\
\hline
\end{tabular}

\section{PROPOSED LUMBAR DISK 3D MODEL CONSTRUCTION}

The proposed lumbar disk model construction method is summarized by the following steps. Given three slices that cut the lumbar inter-vertebral disk that exists between L4-L5 vertebrae in three locations (upper, middle and, lower), (A) extract the region of interest (ROI) from each slice and enhance them by applying some image processing techniques, (B) interpolate the three available slices to generate artificial inter-slices between them, (C) stack the slices anf the artificial slices together in one 3D image, (D) and visualize the $3 \mathrm{D}$ isosurface data using the marching cube algorithm [21] in order to create a 3D mesh.

\subsection{ROI extraction and preprocessing}

The ROI extraction allows the CAD system to work easier because the analysis includes only a small region in the image rather than processing a large detailed image [18]. The areas to be selected is determined by the radiologist's guidance and is then modeled to be detected automatically. In our case, we are interested in the area around the middle of the axial MRI slice. To perform ROI extraction, we used the same method used in previous works $[7,18]$. This method is called the Heuristic ROI extraction, where it is done over two passes; vertical and horizontal extractions. A sample of ROI extraction steps and results are illustrated in Figure 3.

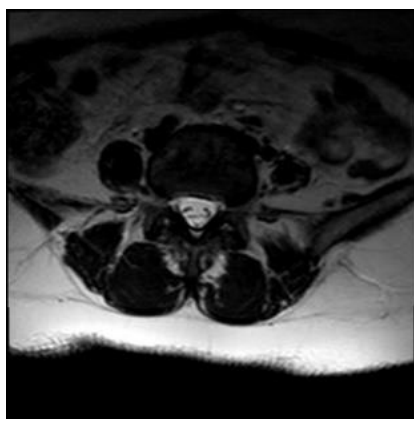

(a)

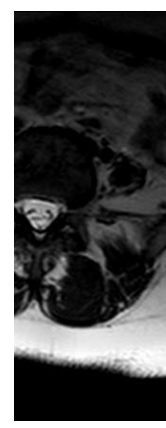

(b)

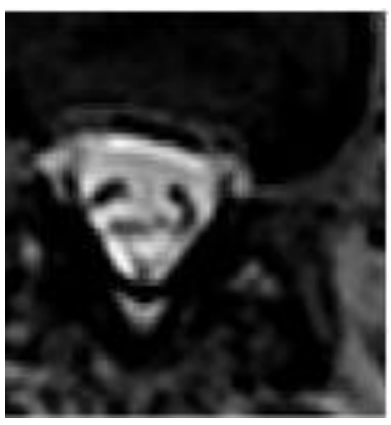

(c)

Figure 3. ROI extraction results, (a) original slice, (b) vertical extraction, (c) final strip of ROI 
After extracting the ROI, we perform some enhancements over the extracted ROI in order to increase the details in the image which increases the accuracy of the next steps. The enhancements that we are interested in aims to increase the details that show the edges that separate the tissues from each other in the stripped ROI. The method that we use in edge sharping is called the unsharp masking [22] in which the image (itself) is subtracted from a blurred (unsharp) version of the image. Unsharp masking produces an edgy image $g(x, y)$ from the input image $f(x, y)$ as in the following equation:

$$
g(x, y)=f(x, y)-f \operatorname{smooth}(x, y)
$$

where fsmooth $(x, y)$ is a smoothed version of the original image $f(x, y)$. Finally, we combine all of this into the equation:

$$
F \operatorname{sharp}(x, y)=f(x, y)+k * g(x, y)
$$

where $k$ is a scaling constant. Logical values of $k$ might be within the range [0,2], the larvger values provides increasing amounts of sharpening. By experimenting with different values of $k$, we set its value to 0.6. We used the Gaussian blurring filter [23] in order to smooth the image. The result of this process is illustrated in Figure 4.

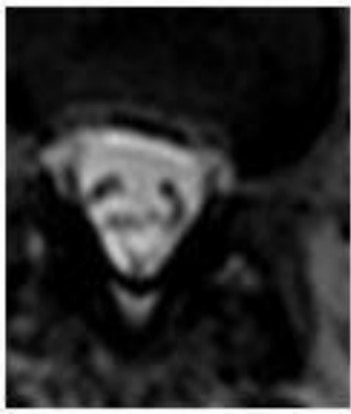

(a)

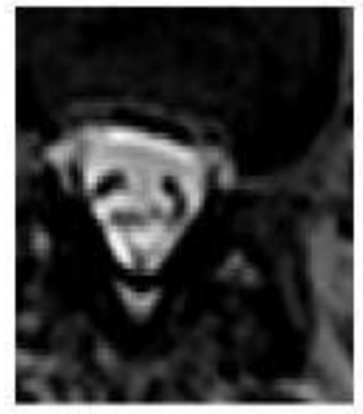

(b)

Figure 4. ROI enhancement results, (a) before enhancement, (b) after enhancement

\subsection{Inter-slice interpolation}

We only have three 2D slices for each disk, where the disk information between these slices is unknown. This problem can be solved by interpolating the available $2 \mathrm{D}$ slices to try to recover some information about the disk area between the available slices. Doing that may lead to producing 3D volume with more details. Interpolation is a method of constructing new data points within the range of a discrete set of data points that are known [24]. This step can be done by a simple linear interpolation technique which is fast, but if the distance between the slices is too much, then the method produces discrepancies in the interpolated slices. Those discrepancies happen because tissues can expand, shrink, bend or even disappear between two successive slices. Let us say that $S 1$ and $S 2$ are two slices that we need to interpolate, a slice $S k$ that is exactly halfway between $S 1$ and $S 2$ is calculated using the following equation:

$$
S k(t=0.5)=(S 1+S 2) / 2
$$

where $t$ is the space between images and it is between 0 and 1 . In general, we can interpolate between at any space $t$ using the following equation:

$$
S(t)=(1-t) * S 1+t * S 2
$$

\subsection{D model construction and visualization}

At this step, we combine the interpolated slices in one single image of four dimensions as a stack. Those four dimensions are $\mathrm{x}^{*} \mathrm{x} * 1 * \mathrm{n}$, where the first two $\mathrm{x}$ are the dimensions of the slices, the third dimension is for the image color data and $\mathrm{n}$ is for the number of interpolated slices. However, since we are dealing with grayscale images the third dimension is no longer needed; so, we can eliminate it to have a 3Darray. 
We only care about the posterior part of the disk in the area that touches the upper edge of the spinal canal and the lower edge of the disk. This region forms only about $25 \%$ of the first of the y-axis. Thus, sub- volume is done by cutting the original 3D array and having only the first $25 \%$ of the voxels. The data we are having now is still voxels, where they are pixels in a volume space [25]. In order to convert volumetric data into a geometrical surface, we have to convert those voxels to triangles; this process is called Triangulation. One of the triangulation techniques used mainly in the field of 3D reconstructing from CT scans or MRI is the Isosurface. Isosurface is a common method in both medical image processing and computer graphics. It is used for visualizing volumes based on the $3 \mathrm{D}$ grids that contain discrete values of a density function [26]. So, the isosurface of a grid for a specific isovalue is the set of whole points that their density equals to that isovalue. It is being used to represent regions of a particular density in a 3D CT or MRI scans, allowing the visualization of internal organs, bones, or other structures.

Different isovalues output different isosurfaces, with less or more data (details). As a rule, the lower isovalue would involve more data in the isosurface. The higher isovalue would drive out noise, but we will obtain fewer data. The common situation is to try different isovalues in order to find which one works best. The isovalues that we are interested in are the values that represent the boundaries such as the boundaries separating between the bone and the soft tissues [27]. Isosurface extraction implementations usually use the Marching Cubes algorithm [25], which return a triangular surface that looks like tessellated surface. Also, the isocaps (ends of isosurface) must be extracted. Isocaps are extracted for the purpose of creating end-caps for isosurfaces of the 3D data, where it places caps at the open ends of the isosurfaces. These triangulated surfaces are sets of faces and vertices, where each triangle is a face and the points on the corners of each face are the vertices.

In order to build a single solid and coherent model, we need to combine those isosurfaces and isocaps in one mesh. In order to build a single solid and coherent model, we need to combine those isosurfaces and isocaps in one polygonal 3D mesh. A polygonal 3D mesh consists of three kinds of mesh elements: vertices, edges, and faces [25]. A vertex is a position or a coordinate in a 3D space with other information such as color, normal vector, and texture coordinates. An edge is a connection between two vertices. A face is a closed group of edges and a triangle face has three edges. To build the mesh, we combine the faces and the vertices resulted from the isosurfaces with those which are extracted from the isocaps. Mesh elements are constructed in a special type of triangulated mesh data structure called Face-Vertex Mesh [28]. It is defined for each face (of degree d); store d references to incident vertices for each vertex, and one reference to its coordinates. Figure 5 shows the model we obtain after combining the isosurface with the isocaps. As mentioned, we need only the first $25 \%$ of the y-axis in our diagnosing process.

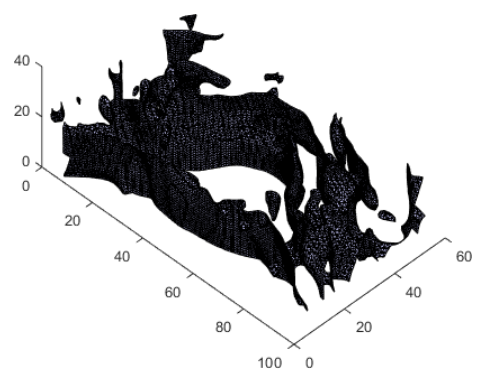

(a)

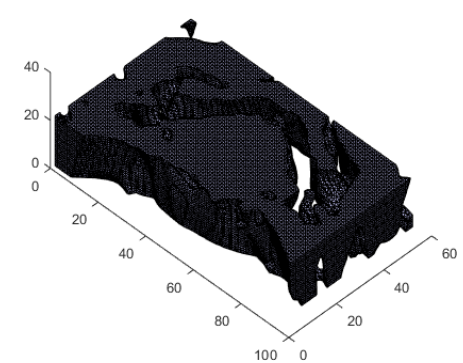

(c)

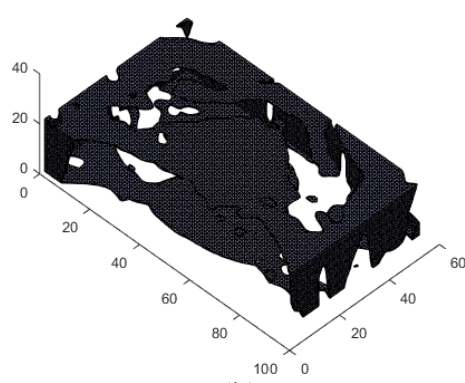

(b)

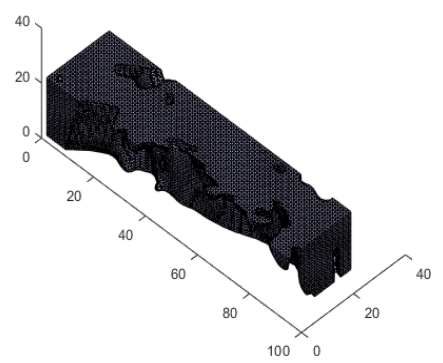

(d)

Figure 5. 3D mesh visualization, (a) isosurface, (b) isocaps, (c) combination result, (d) only $25 \%$ y-axis of the model 


\section{RESULTS}

The proposed 3D model construction method is applied on the 83 cases that we have in the dataset described in section 3. Unfortunately, it is impossible to have a ground truth 3D model that models lumbar disks as they may differ from person to person. For that reason, the evaluation of the cases in our dataset is performed by a radiologist by visualizing only the 3D models rather than analyzing the set of $2 \mathrm{D}$ slices. Our 3D modeling framework is validated by comparing the $2 \mathrm{D}$ diagnosing results versus the $3 \mathrm{D}$ diagnosing results.

There were only four cases in which the radiologist needed to get back to the $2 \mathrm{D}$ images. After investigating these cases, we find that the problem in the construction of the model is caused by some erroneous slices that may result from partial volume overlap between the disk region and the bone of the upper or lower vertebrae. By looking at the numbers, the evaluation shows that the proposed 3D model construction has an accuracy of more than $95 \%$.

\section{CONCLUSION}

In this paper, we presented a simple, robust, and fully automated 3D model construction framework for medical images with a special focus on axial MRI of the lumbar spine. Our framework was tested on a dataset that contains 83 patient cases. We first extracted the ROI, then we enhance the ROI edges, the ROIs from different slices are then interpolated to fill the gaps between the slides, finally, we stacked the images in a 3D matrix to be used in the extraction of the model. The 3D model was evaluated by a radiologist and the evaluation resulted and accuracy of more than $95 \%$. In the future, we hope to be able to use the model in the diagmosing process of intervertebral discs herniation.

\section{REFERENCES}

[1] Ajaz Hussain Mir, "Segmentation of the herniated intervertebral discs," International Journal of Image, Graphics and Signal Processing, vol. 10, no. 6, paper. 31, 2018.

[2] Megha P. Arakeri, G. Ram Mohana Reddy, and Ram Mohana, "An effective and efficient approach to 3d reconstruction and quantification of brain tumor on magnetic resonance images," International Journal of Signal Processing, Image Processing and Pattern Recognition, vol. 6, no. 3, paper. 111, 2013.

[3] Sofia Michopoulou, "Image analysis for the diagnosis of MR images of the lumbar spine," PhD thesis, UCL (University College London), 2011.

[4] Meelis Lootus, “Automated radiological analysis of spinal MRI," PhD thesis, University of Oxford, 2015.

[5] Subarna Ghosh, Vipin Chaudhary, and Gurmeet Dhillon, "Exploring the utility of axial lumbar MRI for automatic diagnosis of intervertebral disc abnormalities," In Medical Imaging 2013: Computer-Aided Diagnosis, International Society for Optics and Photonics, vol. 8670, pp. 86703D, 2013.

[6] Neculai Archip, Robert Rohling, Vincent Dessenne, Pierre-Jean Erard, and Lutz Peter Nolte, "Anatomical structure modeling from medical images," Computer Methods and Programs in Biomedicine, vol. 82, no. 3, pp. 203-215, 2006.

[7] Khaled Alawneh, Mays Al-dwiekat, Mohammad Alsmirat, and Mahmoud Al-Ayyoub, "Computer-aided diagnosis of lumbar disc herniation," In Information and Communication Systems (ICICS), 2015 6th International Conference on, IEEE, pp. 286-291, 2015.

[8] Alomari Raja'S., Jason J. Corso, and Vipin Chaudhary, "Labeling of lumbar discs using both pixel-and object-level features with a two-level probabilistic model," IEEE transactions on medical imaging, vol. 30, no. 1, pp. 1-10, 2011.

[9] Rabia Haq, Rifat Aras, David A. Besachio, Roderick C. Borgie, and Michel A. Audette, "3d lumbar spine intervertebral disc segmentation and compression simulation from MRI using shape-aware models," International journal of computer assisted radiology and surgery, vol. 10, no. 1, pp. 45-54, 2015.

[10] Jessica Benitez Mendieta, "An efficient and semiautomatic segmentation method for 3D surface reconstruc- tion of the lumbar spine from Magnetic Resonance Imaging (MRI), PhD thesis, Queensland University of Technology, 2016.

[11] A. Beulah, T. Sree Sharmila, and V. K. Pramod, "Disc bulge diagnostic model in axial lumbar MR images using intervertebral disc descriptor (idd)," Multimedia Tools and Applications, pp. 1-16, 2018.

[12] Jianhua Yao, Tobias Klinder, and Shuo Li, "Computational Methods and Clinical Applications for Spine Imaging," Springer, 2014.

[13] S. M. Ali, Loay Kadom Abood, and Rabab Saadoon Abdoon, "Automatic technique to produce 3D image for brain tumor of MRI images," Journal of University of Babylon, vol. 22, no. 7, pp. 1896-1907, 2014.

[14] Chethan kumar and Anitha kumari, "3D reconstruction of brain tumor from 2D MRI's using fcm and march-ing cubes," International Journal of Advanced Research in Electronics and Communication Engineering, vol. 3, no. 9, pp. 970-974, 2014.

[15] Christos P. Loizou, Christos Papacharalambous, Giorgos Samaras, Efthyvoulos Kyriacou, Takis Kasparis, Marios Pantziaris, Eleni Eracleous, and Constantinos S. Pattichis, "Brain image and lesions registration and 3d reconstruction in dicom MRI images," In Computer-Based Medical Systems (CBMS), 2017 IEEE 30th International Symposium on, pp. 419-422, 2017. 
[16] Hadia Bashir, Fawad Hussain, and Muhammad Haroon Yousaf, "Smart algorithm for 3D reconstruction and segmentation of brain tumor from MRIs using slice selection mechanism," Smart Computing Review, vol. 5, no. 3, pp. 187-200, 2015.

[17] Volkan Kızılgo“z, Ali Kemal Sivriog־lu, Hasan Aydın, Sunay Sibel Karayol, Can Hakan Yıldırım, and Utku Menderes, "Three-dimensional turbo spin-echo sequence versus conventional two-dimensional turbo spin- echo sequences in the evaluation of lumbar intervertebral discs," The European Research Journal, vol. 4, no. 4, pp. 365-372, 2018.

[18] Mahmoud Al-Ayyoub, Nusaiba Al-Mnayyis, Mohammad A. Alsmirat, Khaled Alawneh, Yaser Jararweh, and Brij B. Gupta, "Sift based roi extraction for lumbar disk herniation cad system from MRI axial scans," Journal of Ambient Intelligence and Humanized Computing, pp. 1-9, 2018.

[19] M. Alsmirat, K. Alawneh, M. Al-Ayyoub, and M. Al-dwiekat, "Building a simulated educational environment for the diagnosis of lumbar disk herniation using axial view MRI scans," Int J Adv Intell Paradig (IJAIP), 2017.

[20] Samah Al-Helo, Raja S. Alomari, Subarna Ghosh, Vipin Chaudhary, Gurmeet Dhillon, Al-Zoubi Mohd B., Hazem Hiary, and Thair M. Hamtini. Compression fracture diagnosis in lumbar: a clinical cad system, "International journal of computer assisted radiology and surgery," vol. 8, no. 3, pp. 461-469, 2013.

[21] William E. Lorensen and Harvey E. Cline, "Marching cubes: A high resolution 3d surface construction algorithm," In ACM siggraph computer graphics, ACM, vol. 21, pp. 163-169, 1987.

[22] Matthew Trentacoste, Ratal Mantiuk, Wolfgang Heidrich, and Florian Dufrot, "Unsharp masking, counter-shading and halos: Enhancements or artifacts?," In Computer Graphics Forum, Wiley Online Library, vol. 31, pp. 555-564, 2012.

[23] Ze-Nian Li, Mark S. Drew, and Jiangchuan Liu, "Fundamentals of multimedia," Springer, 2004.

[24] John Todd, "Basic Numerical Mathematics," International series of numerical mathematics, Birkhauser Basel, 1978.

[25] William J. Schroeder, Kenneth M. Martin, and William E. Lorensen, "The visualization toolkit: an object- oriented approach to 3D graphics; visualize data in 3D-medical, engineering or scientific; build your own applications with C, Tcl, Java ord Python/ Will Schroeder; Ken Martin; Bill Lorensen," Kitware, 2006.

[26] Ran Zask and Matthew N. Dailey, "Rapid 3d visualization of indoor scenes using 3d occupancy grid iso- surfaces," In Electrical Engineering/Electronics, Computer, Telecommunications and Information Tech- nology, 2009. ECTICON 2009. 6th International Conference on, IEEE, vol. 2, pp. 672-675, 2009.

[27] Hamish Carr, Jack Snoeyink, and Michiel Van De Panne, "Flexible isosurfaces: Simplifying and displaying scalar topology using the contour tree," Computational Geometry, vol. 43, no. 1, pp. 42-58, 2010.

[28] M. Botsch, L. Kobbelt, M. Pauly, P. Alliez, and B. Levy, "Polygon Mesh Processing," Ak Peters Series, Taylor \& Francis, 2010. [Online], Available: https://books.google.jo/books?id=8zX-2VRqBAkC. 